\title{
Formation à la conduite
}

\section{François Bellavance Catherine Berthelon}

C IFSTTAR et Éditions NecPlus 2016

Le sur-risque accidentel de certains groupes de conducteurs, plus particulièrement celui des jeunes conducteurs novices, est systématiquement observé dans tous les pays peu importe les programmes de formation et d'accès au permis de conduire mis en place. Le colloque "Formation à la conduite - une vision à $360^{\circ} »$ des $28^{\mathrm{e}}$ Entretiens du Centre Jacques Cartier a réuni les 25 et 26 novembre 2015 à Bruxelles, des représentants d'administrations publiques, d'organisations internationales, des chercheurs, des experts et des formateurs pour échanger et débattre sur les innovations et les bonnes pratiques à adopter pour que les programmes d'éducation à la conduite aient un impact significatif sur le bilan routier.

\section{François Bellavance $(\bowtie)$}

HEC Montréal, Département de sciences de la décision 3000 chemin de la Côte-Sainte-Catherine, Montréal, Quebec, H3T 2A7, Canada e-mail : françois Bellavance@hec.ca

Catherine Berthelon $(\square)$

Ifsttar, Laboratoire Mécanismes d'accidents (LMA), Département Transport Santé Sécurité (TS2) Rédactrice en chef de RTS

(Recherche-Transports-Sécurité)

e-mail : catherine.berthelon@ifsttar.fr
Dans ce cadre, la matrice GDE (Goals for Driver Education) a généré des changements, principalement en Europe, dans la conception et les pratiques de la formation à la conduite où une plus grande attention est maintenant portée sur la compréhension des facteurs de risque et sur les compétences d'autoévaluation de son propre comportement et de ses capacités par l'apprenant. D'autre part, les principes de l'accès graduel à la conduite, surtout adoptés en Amérique du Nord, en Nouvelle-Zélande et en Australie, ont permis de diminuer les accidents des jeunes conducteurs novices en réduisant leur exposition aux risques.

Trois des chercheurs invités à présenter leurs recherches au colloque ont chacun contribué à ce numéro par un article dont l'ensemble présente une revue des éléments clés de l'accès graduel à la conduite et des innovations inspirées de la matrice GDE introduites dans la formation des nouveaux conducteurs en Europe et en Amérique du Nord. Les enseignements de ces approches et les tendances récentes observées dans certains pays amènent deux de ces articles à proposer des modèles cadres de formation à la conduite qui intègrent à la fois l'accès graduel à la conduite et le développement des compétences présentées dans les différents niveaux de la matrice GDE. 
